\title{
Modeling of unsteady MHD free convection flow with radiative heat transfer in a rotating fluid
}

\author{
Harouna Naroua
}

Département de Mathématiques et Informatique, Université Abdou Moumouni, Niamey, Niger; hnaroua@yahoo.com

Received 4 October 2010; revised 5 November 2010; accepted 10 November 2010.

\begin{abstract}
In this paper, a numerical simulation has been carried out on unsteady hydromagnetic free convection near a moving infinite flat plate in a rotating medium. The temperatures involved are assumed to be very high so that the radiative heat transfer is significant, which renders the problem highly non-linear even with the assumption of a differential approximation for the radiative heat flux. A numerical method based on the Nakamura scheme has been employed to obtain the temperature and velocity distributions which are depicted graphically. The effects of the different parameters entering into the problem have been discussed extensively.
\end{abstract}

Keywords: Modeling; Numerical Simulation; Nakamura Scheme;

Unsteady Hydromagnetic Free Convection; Radiative Heat Transfer; Computer Program

\section{INTRODUCTION}

In recent years, an extensive research effort has been directed towards the theory of rotating fluids owing to its numerous applications in cosmical and geophysical fluid dynamics, meteorology and engineering [1]. Batchelor [2] studied the Ekman layer flow on a horizontal plate. The flow past a horizontal plate has also been studied by Debnath [3-5], Puri and Kulshrestha [6], Tokis and Geroyannis [7]. Investigations on the flow past a vertical plate have been carried out by Tokis [8], Kythe and Puri [9]. Though various methods exist for solving fluid flow problems, Naroua et al. [10-11] presented a finite element approach. The problem of thermal radiation has been approached by Ghosh and Pop [12], Raptis and Perdikis [13].

Since high temperature phenomenon abound in solar physics, particularly in astrophysical studies, radiation effects cannot be neglected. This paper therefore incor- porates radiative transfer into the studies, thereby widening the applicability of the results. For an optically thin gas, which is approximated by a transparent medium, the absorption coefficient (which will be assumed constant in the analysis) $\alpha>>1$ and the radiative flux Q satisfies the non-linear differential equation (Cheng [14])

$$
\frac{\partial Q^{\prime}}{\partial z^{\prime}}=4 \sigma \alpha\left(T^{\prime 4}-T_{\infty}^{\prime 4}\right)
$$

where $T^{\prime}$ is the temperature of the fluid, subscript $\infty$ will be used to denote conditions in the undisturbed fluid and $\sigma$ is the Stefan-Boltzmann constant.

\section{MATHEMATICAL ANALYSIS}

Consider the unsteady flow of an electrically-conducting incompressible viscous fluid past a vertical flat plate which moves in its own plane with velocity $\mathrm{U}_{0}$ and rotates about the $\mathrm{z}^{\prime}$ axis with angular velocity $\Omega$. The plate is maintained at $T_{w}^{\prime}\left(T_{w}^{\prime} \square 1\right)$. Following the arguments given by Tokis [8] and employing Eq.1, the governing equations for a transparent medium are as follows:

$$
\begin{gathered}
\frac{\partial u^{\prime}}{\partial t^{\prime}}-2 \Omega v^{\prime}=v \frac{\partial^{2} u^{\prime}}{\partial z^{\prime 2}}-\frac{\sigma_{c}}{\rho} B_{0}^{2} u^{\prime}+g \beta\left(T^{\prime}-T_{\infty}^{\prime}\right) \\
\frac{\partial v^{\prime}}{\partial t^{\prime}}+2 \Omega u^{\prime}=v \frac{\partial^{2} v^{\prime}}{\partial z^{\prime 2}}-\frac{\sigma_{c}}{\rho} B_{0}^{2} v^{\prime} \\
\rho C_{p} \frac{\partial T^{\prime}}{\partial t^{\prime}}=k \frac{\partial^{2} T^{\prime}}{\partial z^{\prime 2}}-4 \sigma \alpha\left(T^{\prime 4}-T_{\infty}^{\prime 4}\right)
\end{gathered}
$$

where $\left(u^{\prime}, v^{\prime}, 0\right)$ are the velocity components, $\mathrm{k}$ is the thermal conductivity, $g$ is the gravitational acceleration, $\sigma_{\mathrm{c}}$ is the electrical conductivity, $v$ is the kinematic viscosity, $\beta_{0}$ is the coefficient of volumetric thermal expansion and $C_{p}$ is the specific heat at constant pressure.

The boundary conditions are:

$$
\begin{aligned}
& u^{\prime}=U_{0}, v^{\prime}=0, T^{\prime}=T_{w}^{\prime} \quad \text { on } z^{\prime}=0 \\
& u^{\prime}=0, v^{\prime}=0, T^{\prime}=T_{\infty}^{\prime} \quad \text { as } z^{\prime} \rightarrow \infty
\end{aligned}
$$


Introducing the following non-dimensional quantities:

$$
\begin{aligned}
& t=\frac{t^{\prime} U_{0}^{2}}{v}, z=\frac{z^{\prime} U_{0}}{v},(u, v)=\frac{\left(u^{\prime}, v^{\prime}\right)}{U_{0}},\left(\theta, \theta_{w}\right)=\left(\frac{\left(T^{\prime}, T_{w}^{\prime}\right)}{T_{\infty}^{\prime}}\right), \\
& E=\frac{\Omega v}{U_{0}^{2}}, \operatorname{Pr}=\frac{\rho v C_{p}}{k}, M^{2}=\frac{\sigma_{c} B_{0}^{2} v}{\rho U_{0}^{2}}, \\
& G r=\frac{g \beta v T_{\infty}^{\prime}}{U_{0}^{3}}, R=\frac{4 \sigma_{c} \alpha v T_{\infty}^{\prime 3}}{\rho C_{p} U_{0}^{2}}
\end{aligned}
$$

The Eqs.2-5 reduce to:

$$
\left\{\begin{array}{l}
\frac{\partial q}{\partial t}+2 i E q=\frac{\partial^{2} q}{\partial z^{2}}-M^{2} q+G r(\theta-1) \\
\operatorname{Pr} \frac{\partial \theta}{\partial t}=\frac{\partial^{2} \theta}{\partial z^{2}}-R\left(\theta^{4}-1\right)
\end{array}\right.
$$

where $\mathrm{q}=\mathrm{u}+\mathrm{iv}$ and

$$
\left\{\begin{array}{cccc}
q=1, & \theta=\theta_{w} & \text { at } & z=0 \\
q=0, & \theta=0 & \text { as } & z \rightarrow \infty
\end{array}\right.
$$

Using $\mathrm{q}=\mathrm{u}+\mathrm{iv}$, the system of Eqs.7,8 becomes:

$$
\left\{\begin{array}{l}
\frac{\partial u}{\partial t}-2 E v=\frac{\partial^{2} u}{\partial z^{2}}-M^{2} u+G r(\theta-1) \\
\frac{\partial v}{\partial t}+2 E u=\frac{\partial^{2} v}{\partial z^{2}}-M^{2} v \\
\operatorname{Pr} \frac{\partial \theta}{\partial t}=\frac{\partial^{2} \theta}{\partial z^{2}}-R\left(\theta^{4}-1\right)
\end{array}\right.
$$

The above system of Eqs.10-12 with boundary conditions (9) has been solved numerically by a computer program using a finite-difference scheme as described by Nakamura [15]. The mesh system is shown in Figure 1.

Eqs.10-12 are coupled non-linear parabolic partial differential equations in $\mathrm{u}, \mathrm{v}$ and $\Theta$. We first discretise them using the backward difference approximation (which is stable) in the time coordinate as shown in Eqs. 13-15:

$$
\left\{\begin{array}{l}
u^{\prime \prime}-\left(M^{2}+\frac{1}{\Delta t}\right) u=G r(1-\theta)-\frac{u_{i, j-1}}{\Delta t}-2 E v \\
v^{\prime \prime}-\left(M^{2}+\frac{1}{\Delta t}\right) v=2 E u-\frac{v_{i, j-1}}{\Delta t} \\
\theta^{\prime \prime}-\left(R \theta^{3}+\frac{\operatorname{Pr}}{\Delta t}\right) \theta=-\frac{\operatorname{Pr}}{\Delta t} \theta_{i, j-1}-R
\end{array}\right.
$$

where $u^{\prime \prime}, v^{\prime \prime}, \theta^{\prime \prime}$ are derivatives with respect to $\mathrm{z}$.

For the sake of simplicity, we write:

$$
\begin{aligned}
& B_{2}=1 ; \quad B_{1}=0 ; \quad B_{0}=-\left(M^{2}+\frac{1}{\Delta t}\right) ; \\
& C_{2}=1 ; \quad C_{1}=0 ; \quad C_{0}=-\left(M^{2}+\frac{1}{\Delta t}\right) ; \\
& D_{2}=1 ; \quad D_{1}=0 ; \quad D_{0}=-\left(R \theta^{3}+\frac{\operatorname{Pr}}{\Delta t}\right)
\end{aligned}
$$

Using the above formulation, Eqs.13-15 take the form:

$$
\left\{\begin{array}{l}
B_{2} u_{i, j}^{\prime \prime}+B_{1} u_{i, j}^{\prime}+B_{0} u_{i, j}=G r\left(1-\theta_{i, j}\right)-\frac{1}{\Delta t} u_{i, j-1}-2 E v_{i, j} \\
C_{2} v_{i, j}^{\prime \prime}+C_{1} v_{i, j}^{\prime}+C_{0} v_{i, j}=2 E u_{i, j}-\frac{1}{\Delta t} v_{i, j-1} \\
D_{2} \theta_{i, j}^{\prime \prime}+D_{1} \theta_{i, j}^{\prime}+D_{0} \theta_{i, j}=-\frac{\operatorname{Pr}}{\Delta t} \theta_{i, j-1}-R
\end{array}\right.
$$

Using the central difference scheme which is unconditionally stable, Eqs.16-18 reduce to:

$$
\left\{\begin{array}{l}
B_{2}\left(\frac{u_{i+1, j}-2 u_{i, j}+u_{i-1, j}}{(\Delta z)^{2}}\right)+B_{1}\left(\frac{u_{i+1, j}-u_{i-1, j}}{2 \Delta z}\right)+B_{0} u_{i, j}=G r\left(1-\theta_{i, j}\right)-\frac{1}{\Delta t} u_{i, j-1}-2 E v_{i, j} \\
C_{2}\left(\frac{v_{i+1, j}-2 v_{i, j}+v_{i-1, j}}{(\Delta z)^{2}}\right)+C_{1}\left(\frac{v_{i+1, j}-v_{i-1, j}}{2 \Delta z}\right)+C_{0} v_{i, j}=2 E u_{i, j}-\frac{1}{\Delta t} v_{i, j-1} \\
D_{2}\left(\frac{\theta_{i+1, j}-2 \theta_{i, j}+\theta_{i-1, j}}{(\Delta z)^{2}}\right)+D_{1}\left(\frac{\theta_{i+1, j}-\theta_{i-1, j}}{2 \Delta z}\right)+D_{0} \theta_{i, j}=-\frac{\operatorname{Pr}}{\Delta t} \theta_{i, j-1}-R
\end{array}\right.
$$




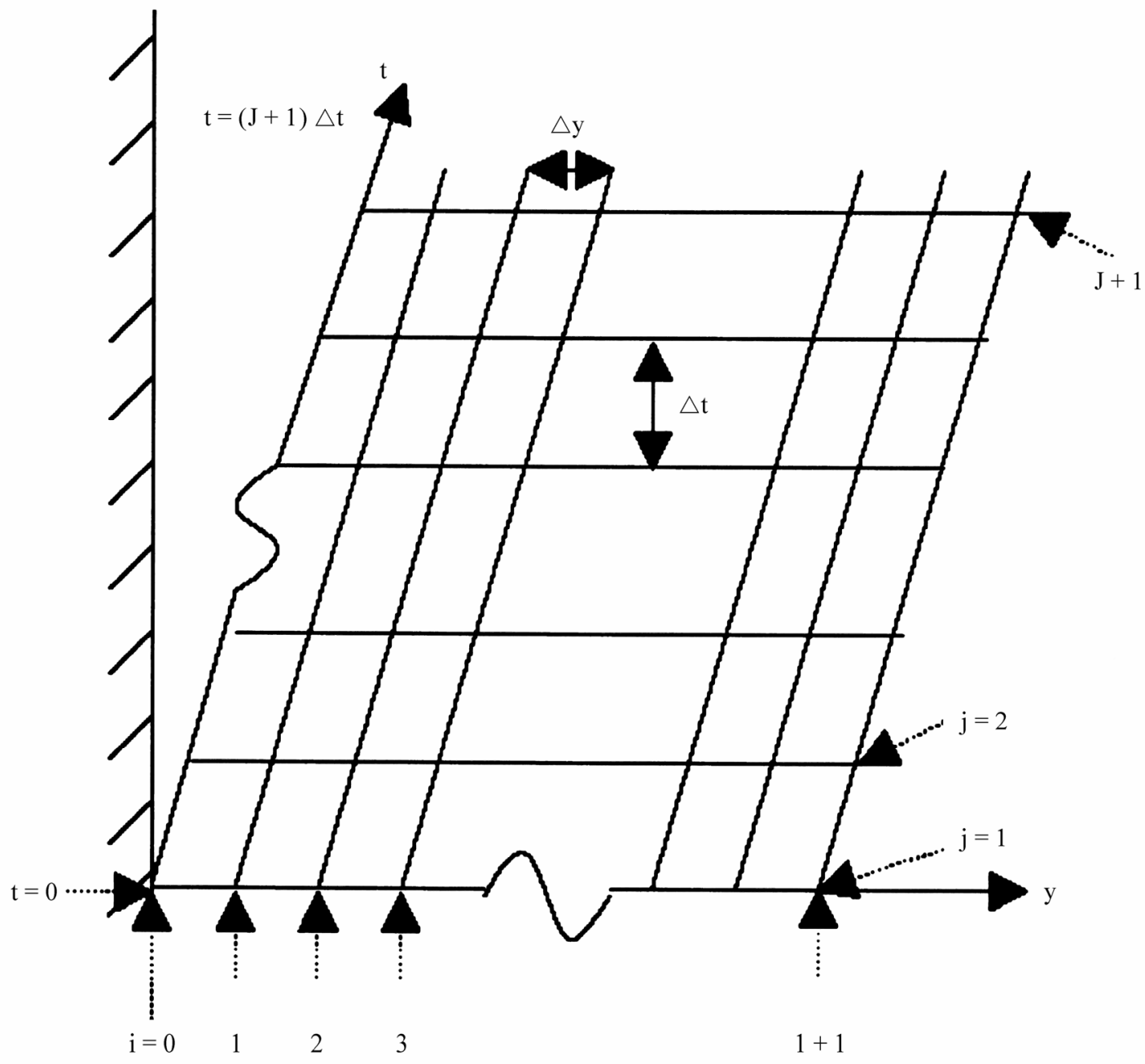

Figure 1. Mesh system.

Since $B_{1}=C_{1}=D_{1}=0$, at time step $\mathrm{j}+1$, Eqs.19-21 reduce to:

$$
\left\{\begin{array}{l}
\left(\frac{B_{2}}{(\Delta z)^{2}}\right) \cdot u_{i-1, j+1}+\left(B_{0}-\frac{2 B_{2}}{(\Delta z)^{2}}\right) \cdot u_{i, j+1}+\left(\frac{B_{2}}{(\Delta z)^{2}}\right) \cdot u_{i+1, j+1}=G r\left(1-\theta_{i, j+1}\right)-\frac{1}{\Delta t} u_{i, j}-2 E v_{i, j+1} \\
\left(\frac{C_{2}}{(\Delta z)^{2}}\right) \cdot v_{i-1, j+1}+\left(C_{0}-\frac{2 C_{2}}{(\Delta z)^{2}}\right) \cdot v_{i, j+1}+\left(\frac{C_{2}}{(\Delta z)^{2}}\right) \cdot v_{i+1, j+1}=2 E u_{i, j+1}-\frac{1}{\Delta t} v_{i, j} \\
\left(\frac{D_{2}}{(\Delta z)^{2}}\right) \theta_{i-1, j+1}+\left(D_{0}-\frac{2 D_{2}}{(\Delta z)^{2}}\right) \cdot \theta_{i, j+1}+\left(\frac{D_{2}}{(\Delta z)^{2}}\right) \cdot \theta_{i+1, j+1}=-\frac{\operatorname{Pr}}{\Delta t} \theta_{i, j}-R
\end{array}\right.
$$

Eqs.22-24 cannot be solved individually for each grid point $i$. The equations for all the grid points must be solved simultaneously. The set of equations for $i=$
$1,2, \ldots \ldots \ldots . . .$. I forms a tridiagonal system of equations as described by Nakamura [15] and shown in Eqs.25-27. 


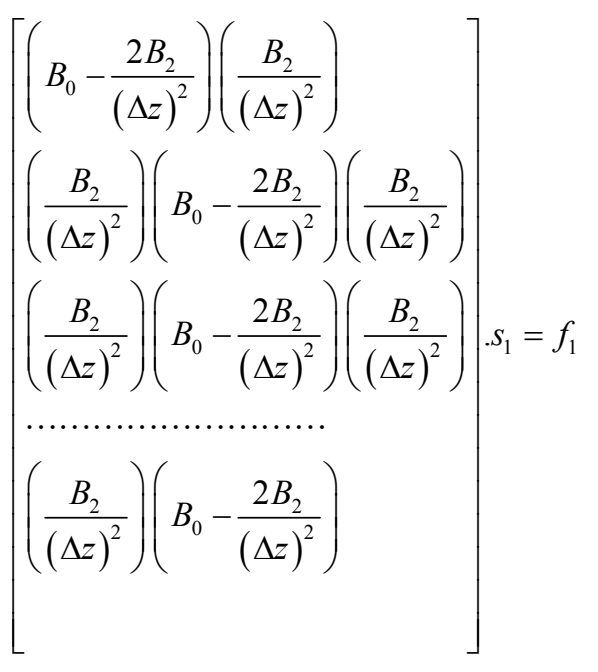

where $s_{1}=\left[\begin{array}{c}u_{1, j+1} \\ u_{2, j+1} \\ u_{3, j+1} \\ \cdots \cdots \\ u_{i, j+1} \\ \cdots \ldots \\ u_{I, j+1}\end{array}\right]$ and

$f_{1}=\left[\begin{array}{l}G r\left(1-\theta_{1, j+1}\right)-\frac{1}{\Delta t} u_{1, j}-2 E v_{1, j+1} \\ G r\left(1-\theta_{2, j+1}\right)-\frac{1}{\Delta t} u_{2, j}-2 E v_{2, j+1} \\ G r\left(1-\theta_{3, j+1}\right)-\frac{1}{\Delta t} u_{3, j}-2 E v_{3, j+1} \\ \cdots \ldots \ldots \ldots \ldots \ldots \ldots \ldots \ldots \ldots \ldots \ldots \ldots \ldots \ldots \ldots \ldots \\ G r\left(1-\theta_{I, j+1}\right)-\frac{1}{\Delta t} u_{I, j}-2 E v_{I, j+1}\end{array}\right]$

$$
\left[\begin{array}{l}
\left(C_{0}-\frac{2 C_{2}}{(\Delta z)^{2}}\right)\left(\frac{C_{2}}{(\Delta z)^{2}}\right) \\
\left(\frac{C_{2}}{(\Delta z)^{2}}\right)\left(C_{0}-\frac{2 C_{2}}{(\Delta z)^{2}}\right)\left(\frac{C_{2}}{(\Delta z)^{2}}\right) \\
\left(\frac{C_{2}}{(\Delta z)^{2}}\right)\left(C_{0}-\frac{2 C_{2}}{(\Delta z)^{2}}\right)\left(\frac{C_{2}}{(\Delta z)^{2}}\right) \\
\cdots \ldots \ldots \ldots \ldots \ldots \ldots \ldots \ldots \ldots . \cdots \\
\left(\frac{C_{2}}{(\Delta z)^{2}}\right)\left(C_{0}-\frac{2 C_{2}}{(\Delta z)^{2}}\right)
\end{array}\right] \cdot f_{2}=f_{2}
$$

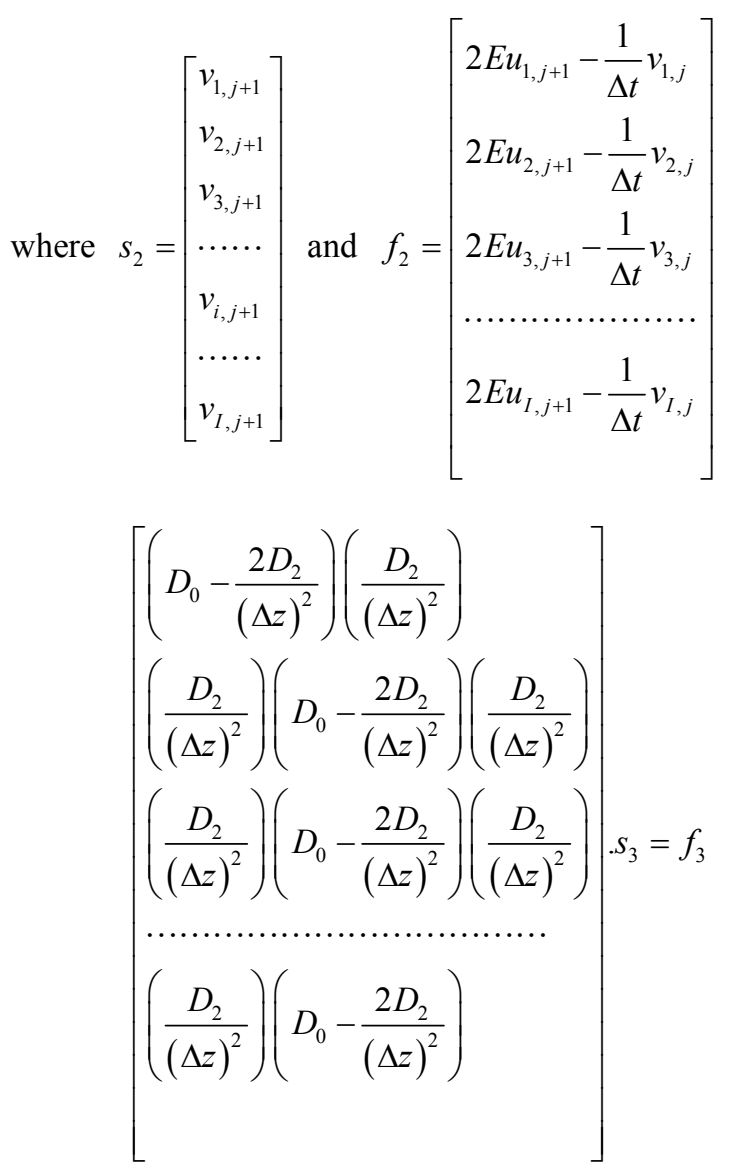

$$
\text { where } s_{3}=\left[\begin{array}{c}
\theta_{1, j+1} \\
\theta_{2, j+1} \\
\theta_{3, j+1} \\
\cdots \ldots \\
\theta_{i, j+1} \\
\cdots \ldots \\
\theta_{I, j+1}
\end{array}\right] \text { and } f_{3}=\left[\begin{array}{c}
-\frac{\operatorname{Pr}}{\Delta t} \theta_{1, j}-R \\
-\frac{\operatorname{Pr}}{\Delta t} \theta_{2, j}-R \\
-\frac{\operatorname{Pr}}{\Delta t} \theta_{3, j}-R \\
\ldots \ldots \ldots \ldots . . \\
-\frac{\operatorname{Pr}}{\Delta t} \theta_{i, j}-R \\
\cdots \ldots \ldots \ldots . . \\
-\frac{\operatorname{Pr}}{\Delta t} \theta_{I, j}-R
\end{array}\right]
$$

For each time step, the system of Eqs.25-27 requires an iterative procedure due to the presence of non-linear coefficients. Successive substitution and iteration are continuously executed for each time step until convergence is reached.

\section{DISCUSSION OF RESULTS}

To get a physical insight into the problem and for the 
purpose of discussing the results, numerical calculations have been carried out for the temperature and velocity profiles and are displayed in Figures 2-6. The velocity profiles are examined for the cases $\mathrm{Gr}>0$ and $\mathrm{Gr}<0$. $\mathrm{Gr}>0(=10)$ is used for the case when the flow is in the presence of cooling of the plate by free convection currents. $\mathrm{Gr}<0(=-10)$ is used for the case when the flow is in the presence of heating of the plate by free convection currents.

From Figure 2, we observe that:

1) The temperature profile decreases far away from the plate. The decrease is greater for a Newtonian fluid than it is for a non-Newtonian fluid ( $\Theta$ decreases with $\mathrm{Pr}$ ).

2) The temperature profile decreases due to an increase in the radiation parameter $\mathrm{R}$ whereas it increases due to an increase in the time $t$.

From Figures 3,4, we observe that:

1) For the case when $\mathrm{Gr}>0(=10)$, in the presence of cooling of the plate by free convection currents, the primary velocity profile $(\mathrm{u})$ increases due to an increase in the time $\mathrm{t}$ and the rotation parameter $\mathrm{E}$; conversely it decreases due to an increase in the Prandtl number Pr and the Magnetic parameter M. There is an insignificant change in the primary velocity profile due to an increase in the radiation parameter $\mathrm{R}$.

2) For the case when Gr $<0(=-10)$, in the presence of heating of the plate by free convection currents, the primary velocity profile $(\mathrm{u})$ increases due to an increase in the time $t$, the Prandtl number Pr and the rotation parameter E; conversely it decreases due to an increase in the Magnetic parameter $M$. There is also an insignificant change in the primary velocity profile due to an increase in the radiation parameter $\mathrm{R}$.

From Figures 5,6, we observe that:

1) Both in the presence of cooling of the plate $(\mathrm{Gr}>0)$ and in the presence of heating of the plate by free convection currents $(\mathrm{Gr}<0)$, there is an insignificant change in the secondary velocity profile $(v)$ due to an increase in the radiation parameter $\mathrm{R}$ whereas it decreases due to an increase in the time $t$, the magnetic parameter $M$ and the rotation parameter $\mathrm{E}$.

2) The secondary velocity profile (v) rises in the presence of cooling of the plate $(\mathrm{Gr}>0)$ and falls in the presence of heating of the plate by free convection currents

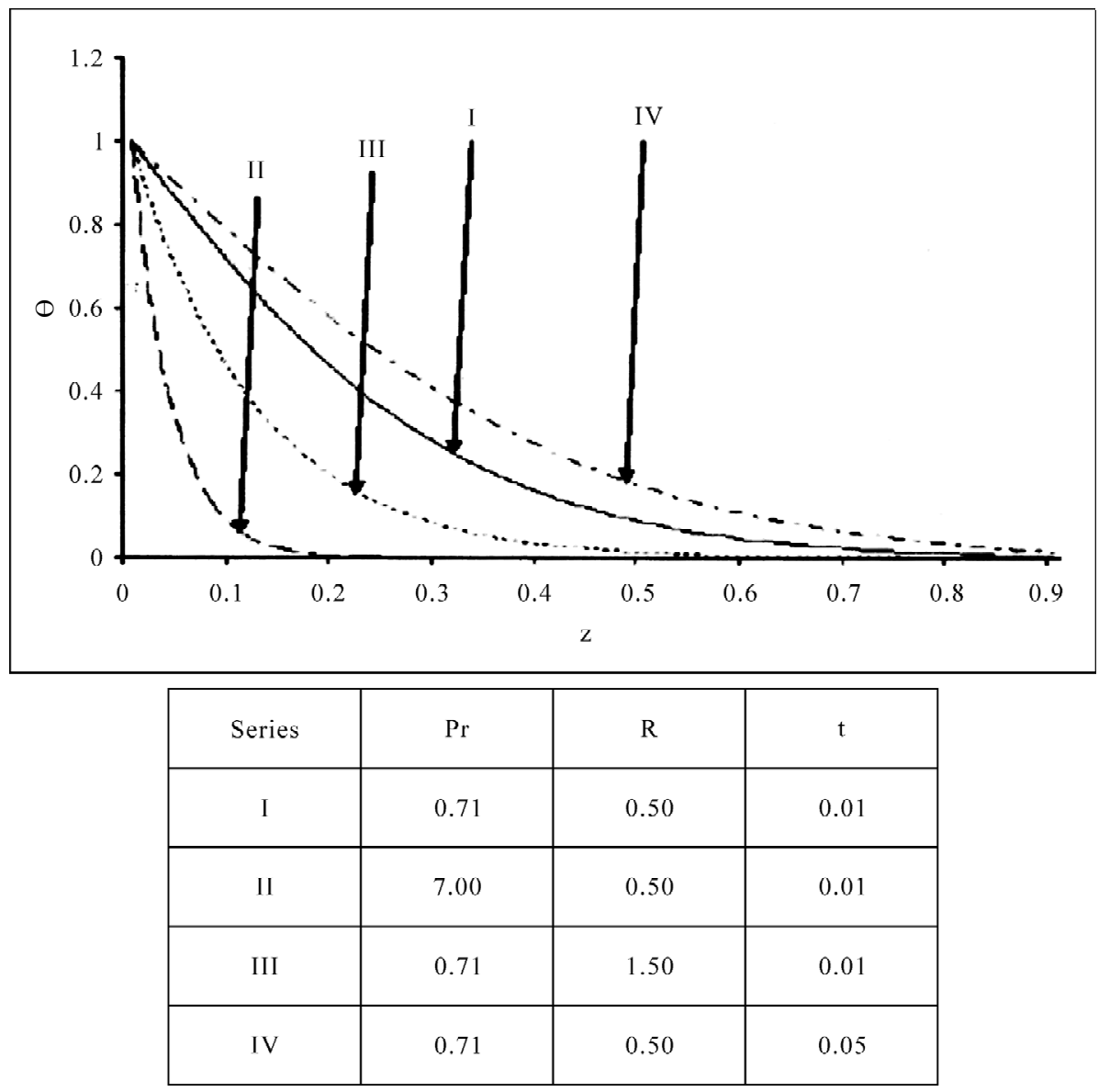

Figure 2. Temperature $(\Theta)$ distribution. 


\begin{tabular}{|l|l|l|l|l|l|}
\hline & \\
\hline
\end{tabular}

Figure 3. Primary velocity (u) distribution for $\mathrm{Gr}=10$.

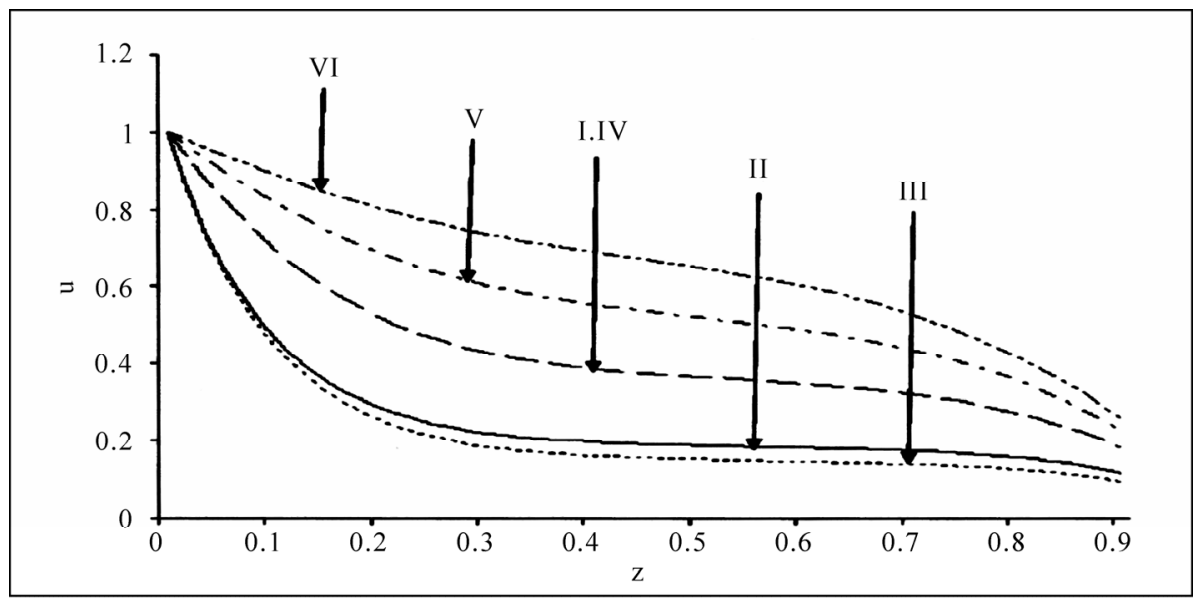

\begin{tabular}{|c|c|c|c|c|c|}
\hline Series & $\operatorname{Pr}$ & $\mathrm{M}$ & $\mathrm{R}$ & $\mathrm{E}$ & $\mathrm{t}$ \\
\hline I & 0.71 & 1.00 & 0.50 & 0.10 & 0.01 \\
\hline II & 7.00 & 1.00 & 0.50 & 0.10 & 0.01 \\
\hline III & 0.71 & 5.00 & 0.50 & 0.10 & 0.01 \\
\hline IV & 0.71 & 1.00 & 1.50 & 0.10 & 0.01 \\
\hline V & 0.71 & 1.00 & 0.50 & 0.50 & 0.01 \\
\hline VI & 0.71 & 1.00 & 0.50 & 0.10 & 0.05 \\
\hline
\end{tabular}

Figure 4. Primary velocity $(\mathrm{u})$ distribution for $\mathrm{Gr}=-10$. 


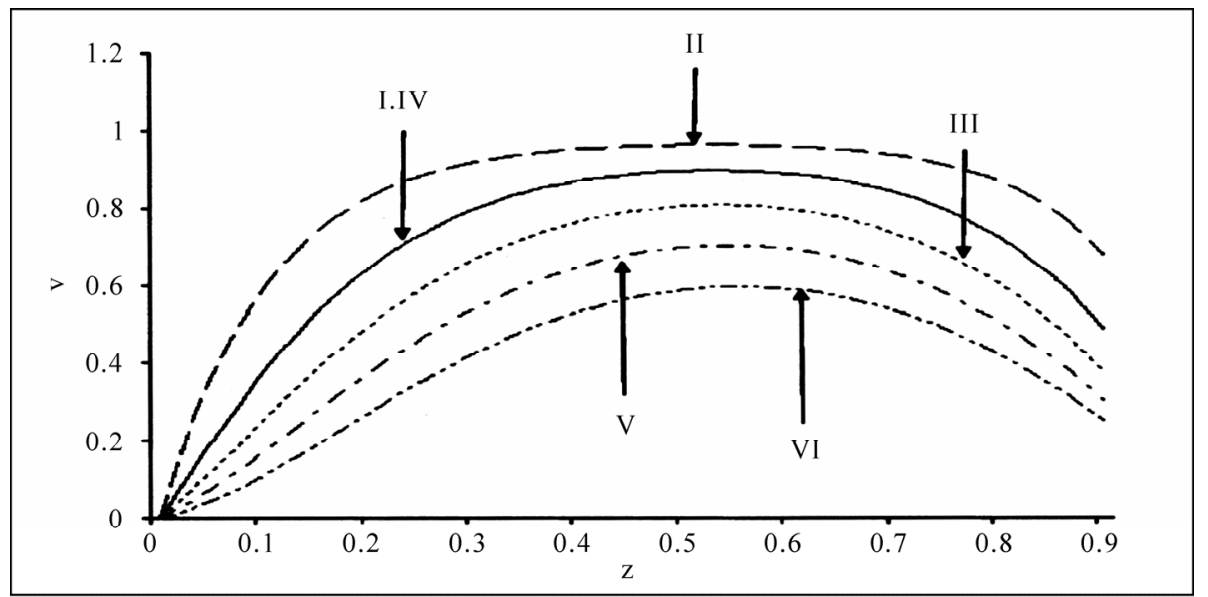

\begin{tabular}{|c|c|c|c|c|c|}
\hline Series & $\mathrm{Pr}$ & $\mathrm{M}$ & $\mathrm{R}$ & $\mathrm{E}$ & $\mathrm{t}$ \\
\hline I & 0.71 & 1.00 & 0.50 & 0.10 & 0.01 \\
\hline II & 7.00 & 1.00 & 0.50 & 0.10 & 0.01 \\
\hline III & 0.71 & 5.00 & 0.50 & 0.10 & 0.01 \\
\hline IV & 0.71 & 1.00 & 1.50 & 0.10 & 0.01 \\
\hline V & 0.71 & 1.00 & 0.50 & 0.50 & 0.01 \\
\hline VI & 0.71 & 1.00 & 0.50 & 0.10 & 0.05 \\
\hline
\end{tabular}

Figure 5. Secondary velocity (v) distribution for $\mathrm{Gr}=10$.

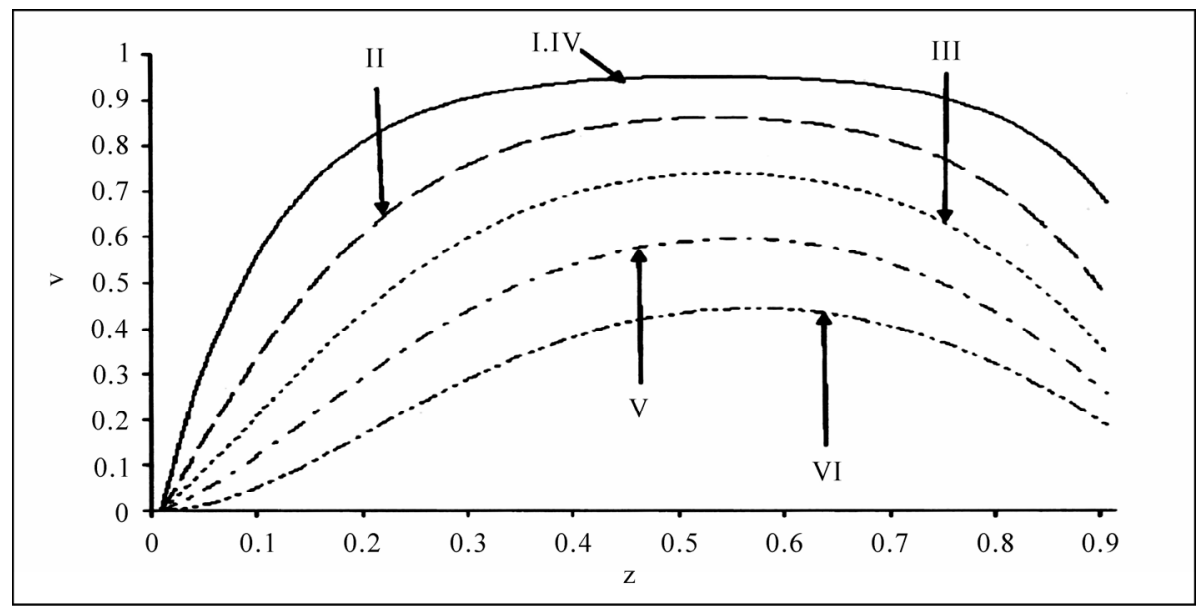

\begin{tabular}{|c|c|c|c|c|c|}
\hline Series & Pr & M & $\mathrm{R}$ & $\mathrm{E}$ & $\mathrm{t}$ \\
\hline I & 0.71 & 1.00 & 0.50 & 0.10 & 0.01 \\
\hline II & 7.00 & 1.00 & 0.50 & 0.10 & 0.01 \\
\hline III & 0.71 & 5.00 & 0.50 & 0.10 & 0.01 \\
\hline IV & 0.71 & 1.00 & 1.50 & 0.10 & 0.01 \\
\hline V & 0.71 & 1.00 & 0.50 & 0.50 & 0.01 \\
\hline VI & 0.71 & 1.00 & 0.50 & 0.10 & 0.05 \\
\hline
\end{tabular}

Figure 6. Secondary velocity (v) distribution for $\mathrm{Gr}=-10$. 
$(\mathrm{Gr}<0)$ due to an increase in Pr.

\section{REFERENCES}

[1] Greenspan, H.P. (1968) The theory of rotating fluids. Cambridge University Press, UK.

[2] Batchelor, G.K. (1967) An introduction to fluid dynamics. Cambridge University Press, UK.

[3] Debnath, L. (1972) On unsteady magnetohydrodynamic boundary layers in a rotating flow. Zeitschrift für Angewandte Mathematik und Mechanik, 52, 623-626.

[4] Debnath, L. (1974) On the unsteady hydromagnetic boundary layer flow induced by torsional oscillations of a disk. Plasma Physics, 16, 1121-1128.

[5] Debnath, L. (1975) Exact solutions of the unsteady hydrodynamic and hydromagnetic boundary layer equations in a rotating fluid system. Zeitschrift für Angewandte Mathematik und Mechanik, 55, 431-438.

[6] Puri, P. and Kulshrestha, P.K. (1976) Unsteady hydromagnetic boundary layer in a rotating medium. Journal of Applied Mechanics, Transactions of the A.S.M.E., 98, 205-208.

[7] Tokis, J.N. and Geroyannis, V.S. (1981) Unsteady hydromagnetic rotating flow near an oscillating plate. $A s-$ trophysics and Space Science, 75, 393-405.

[8] Tokis, J.N. (1988) Free convection and mass transfer effects on the magnetohydrodynamic flows near a mov-

\section{NOMENCLATURE}

$\mathrm{u}$ : the non-dimensional primary velocity

$\mathrm{v}$ : the non-dimensional secondary velocity

$\Theta:$ the non-dimentional temperature

g: the gravitational acceleration

$\beta$ : the volumetric coefficient of thermal expansion

$\mathrm{k}$ : the thermal conductivity

$\sigma_{\mathrm{c}}$ : the electric conductivity

$v$ : the kinematic coefficient of viscosity of the fluid ing plate in a rotating medium. Astrophysics and Space Science, 144, 291-301.

[9] Kythe, P.K. and Puri, P. (1988) Unsteady MHD free-convection oscillatory flow on a porous plate in a rotating medium. Astrophysics and Space Science, 149, 107-114.

[10] Naroua, H. (2007) A computational solution of hydromagnetic-free convective flow past a vertical plate in a rotating heat-generating fluid with Hall and ion-slip currents. International Journal for Numerical Methods in Fluids, 53, 1647-1658.

[11] Naroua, H., Takhar, H.S. and Slaouti, A. (2006) Computational challenges in fluid flow problems: A MHD Stokes problem of convective flow from a vertical infinite plate in a rotating fluid. European Journal of Scientific Research, 13, 101-112.

[12] Ghosh, S.K. and Pop, I. (2007) Thermal radiation of an optically thick gray gas in the presence of indirect natural convection. International Journal of Fluid Mechanics Research, 34, 515-520.

[13] Raptis, A. and Perdikis, C. (2003) Thermal radiation of an optically thin gray gas. International Journal of Applied Electromagnetics and Mechanics, 8, 131-134.

[14] Cheng, P. (1964) Two-dimensional radiating gas flow by a moment method. AIAA Journal, 2, 1662-1664.

[15] Nakamura, S. (1991) Applied numerical methods with software. Prentice-Hall International Editions, USA.
$\mathrm{B}_{0}$ : the coefficient of volumetric thermal expansion

$\mathrm{Cp}$ : the specific heat at constant pressure

E: the rotation parameter

Pr: the Prandtl number

$\mathrm{M}^{2}$ : the magnetic field parameter

Gr: the free convection parameter

$\mathrm{R}$ : the radiation parameter

$\rho: \quad$ the density of the fluid 